WWW.revistadyo.com

\title{
El modelo de negocio como brújula en entornos dinámicos: el caso de Amadeus El modelo de negocio como brújula en entornos dinámicos: el caso de Amadeus
}

Fernando E. García-Muiña, Rocío González-Sánchez y Sonia Medina-Salgado

Departamento de Economía de la Empresa (Administración, Dirección y Organización) Universidad Rey Juan Carlos. fernando.muina@urjc.es, rocio.gonzalez@urjc.es, sonia.medina@urjc.es

Fecha de recepción: | 1-7-2014

Fecha de aceptación: 4-11-2014

Resumen: En el marco de la Teoría de Recursos y Capacidades, el modelo de negocio es una herramienta estratégica de gran utilidad ante el dinamismo del entorno turístico. Por ello, en este trabajo se analiza el uso que Amadeus, uno de los líderes tecnológicos en la intermediación turística, hace de esta herramienta, reconociendo su lógica dominante y ofreciendo una visión holística.

Este modelo descansa sobre una fuerte inversión de recursos humanos y tecnológicos que le permite desarrollar capacidades de innovación, relaciones estables con clientes y un fuerte reconocimiento de marca. No obstante, se han detectado aspectos de mejora en el futuro, como en la apropiación de los beneficios.

Palabras clave: modelo de negocio; recursos y capacidades, creación de valor, intermediación turística y Amadeus.

\begin{abstract}
Given the dynamic conditions of the tourism environment, the ability of firms to both analyze it and adapt to its evolution is determinant, not only for firm survival but also for creating sustainable competitive advantages and value creation. In addition, the tourism industry has been one of the great forgotten in studying the role of resources and capabilities. Taking into account the assumptions of the Resource Based View, the business model is a useful strategic tool for these objectives.

Therefore, we offer a definition for business model and a description of its main dimensions -content, structure and governance mechanisms; in addition, in this paper it is analyzed how Amadeus, a technology leader in the field of tourism intermediation, is facing these changes. Technological innovation distribution became a strategic factor in the tourism sector in general, and airlines in particular. The analysis of these dimensions let us recognize the dominant logic of Amadeus' model and provide a holistic view of the company, contributing to better understand how the organization works, how resources and core competencies are employed through its entire value chain and, how the company redefines its operations when necessary.

It is considered that the business model of Amadeus is virtuous, among other reasons, the complementarity between the different capabilities that make the structure dimension; It rests on a heavy investment in human and technological resources that allows the firm to develop innovation capabilities, stable relationships with customers and strong brand recognition. However, we have detected an issue that will demand special attention in the future: the appropriation of rents; in spite of the positive evolution of its revenues and results, profitability does not show the same trend.
\end{abstract}

Keywords: business model, resources and capabilities, value creation, tourism intermediation and Amadeus.

\section{Introducción: la intermediación turística y la innovación tecnológica}

La innovación juega un papel determinante en el actual contexto de dinamismo y globalización que caracteriza el turismo y las empresas que comercializan sus bienes y servicios (Calero García, Parra López y Santana Talavera, 20 I 0; Spencer, Buhalis y Moital, 2012; Radulovi_, 2013; Thomas y Wood, 2014). En este sentido, la significativa implantación de nuevas tecnologías ha cambiado la forma en que las empresas realizan sus actividades en el sector (Buhalis y Licata, 2002; Ho y Lee, 2007), permitiendo agilizar sus procesos organizativos $y$, de esta manera, incrementando el valor que proporcionan los productos ofrecidos. Consecuentemente, la estructura del sector y las estrategias que en él se llevan a cabo, deben adaptarse a un turismo globalizado y con nuevas reglas en sus cadenas de creación valor (Porter, 200 I; Buhalis y Law, 2008; Spencer et al., 20 I2; Llach, Marimon, Alonso-Almeida y Bernardo, 20।3).

Ante este entorno de cambios continuos, las estrategias de las empresas turísticas deben permitir generar y gestionar satisfactoriamente nuevos recursos intangibles y capacidades (Nieves y Haller, 20 I 4; Thomas y Wood, 20I4), de tal forma que alcancen el éxito al incorporarlos a sus tecnologías, bienes o servicios, de acuerdo con los postulados de la Teoría de Recursos y Capacidades (Barney, 1991). Esta 
toma de decisiones y el análisis de sus consecuencias conforman el conocido como modelo de negocio, herramienta cuyo empleo permitirá a las organizaciones comprender más fácilmente qué variables contribuyen a mejorar su nivel de competitividad y crear valor a partir de su función empresarial (Bradley, 1997; Casadesus-Masanell y Ricart, 2007).

En este sentido, la atención de la Dirección de la empresa turística no debe centrarse sólo en la posesión de determinados recursos, debe considerar también la utilización que la organización haga de ellos, a través de estándares o procesos de uso, a la hora de obtener ventajas competitivas y rentas superiores a sus competidores (Tsoukas, 2005; Calero García et al., 2010 ; Nieves y Haller, 2014). Estos procesos deberán comprender los distintos tipos de recursos que la empresa turística posee -los recursos físicos y financieros, tales como la localización geográfica o los excedentes financieros; los recursos intangibles organizativos, entre ellos tecnología y la marca; y los recursos humanos, con un importante peso en el sector, fundamentalmente el know-how y las habilidades de los trabajadores de la empresa- (Barney, 1991; Koch y McGrath, 1996).

La innovación tecnológica convirtió la distribución en un factor estratégico en el sector turístico en general, y en de las compañías aéreas en particular, con la aparición en los años 60 del pasado siglo de los denominados Sistemas Centrales de Reservas de las compañías aéreas (CRS), que permitieron alcanzar importantes barreras de entrada gracias a economías de escala y experiencia (Granados, Kauffman, King, 2008). Los CRS proporcionaban información centralizada sobre programación, disponibilidad y tarifas de los asientos de las distintas clases en un vuelo y a la que las agencias de viaje tuvieron acceso en los años 70 (Radulović, 2013). Así, las agencias lograron agilizar y hacer más eficiente el proceso completo de reserva del producto aéreo al poder acceder directamente a los sistemas de reservas de las distintas compañías a través de la firma de acuerdos a largo plazo (Granados et al., 2008). Sin embargo, la consideración aislada de los productos turísticos no admitía una gestión global de la variedad de productos y servicios que un viajero requería en la organización de su viaje. Por ello, una década después, a partir de la infraestructura tecnológica desarrollada por las empresas propietarias de los sistemas, se derivó hacia los actuales Sistemas Globales de Distribución (GDS), que incluían una mayor cartera de productos y soluciones tecnológicas y, en consecuencia, una distribución más adaptada a las necesidades de los abonados. De esta manera, los minoristas y mayoristas de viajes comenzaron a ofrecer un producto turístico integrado que conectaba a una gran variedad de proveedores y viajeros (Sismanidou, Palacio y Tafur, 2009; Radulović, 201 3). Tradicionalmente, las empresas propietarias de estos sistemas de distribución electrónica han disfrutado de una posición privilegiada y protegida en la distribución de viajes, debido a la importante inversión que se requeriría por parte de los nuevos participantes, y los elevados costes de cambio y aprendizaje para poner a prueba nuevas formas de distribución (Granados et al., 2008).

Sin embargo, actualmente esta barrera de entrada tecnológica se ha visto amenazada, ya que «el advenimiento de Internet y de las tecnologías de fuentes abiertas han facilitado una desintermediación en la distribución con la promesa de una reducción de costes» (Sismanidou et al., 2009, p. 25 I) desconocidas hasta este momento. Amparados por este medio universal e interactivo, los proveedores de servicios turísticos desarrollaron aplicaciones de comercio electrónico que permitieron a los usuarios acceder directamente a sus sistemas de reservas, logrando una relación más cercana con ellos (Buhalis y Licata, 2002). Podemos hablar, en consecuencia, de una reestructuración de las actividades tradicionales, que está estableciendo un nuevo mapa de relaciones entre los agentes implicados (Buhalis y Law, 2008; Flores Alberto, Salazar Niebla y Santana Turégano, 20 I I; Llach et al., 20।3). En esta línea, la primacía del negocio de los GDS se está viendo afectado por la llegada nuevos actores -GDS New Entrant (GNEs)que ofrecen, a un menor coste, soluciones tecnológicas alternativas para la realización de reservas (Buhalis y Licata, 2002; Granados et al., 2008; Sismanidou et al., 2009).

Pero esta desintermediación no es siempre posible (Flores Alberto et al., 20 I I), al menos en su totalidad y en sentido estricto, para muchos de los proveedores de productos y servicios turísticos, al no disponer de los conocimientos ni la base tecnológica necesaria para el desarrollo de herramientas tecnológicas que se ajusten a sus necesidades. Ello se traducirá en la búsqueda y establecimiento de relaciones con un intermediario que actúe como un proveedor tecnológico. Esta situación ha obligado a las empresas propietarias de los GDS, que habían realizado su trabajo en un mercado de oligopolio, intensivo en información y en un nivel de intermediación mayorista, a diversificar y completar su cartera de negocio para ofrecer soluciones adaptadas a las nue- 
vas necesidades de sus clientes y seguir manteniendo su primacía en el sector (Alamdari y Mason, 2006). En resumen, se trataría de complementar su papel como distribuidor con el de posibilitador, proporcionando una mayor interconectividad entre los agentes implicados (Buhalis y Licata, 2002).

Partiendo de estas premisas, el modelo de negocio se convierte en un instrumento muy útil para aplicar los argumentos generales de la Teoría de Recursos a una realidad empresarial concreta, a partir de la gestión de sus recursos y capacidades estratégicos así como su influencia sobre la creación y sostenimiento de las ventajas competitivas. Además, el sector turístico ha sido uno de los grandes olvidados en el estudio del papel de los recursos y capacidades frente a otros sectores, como el manufacturero o los intensivos en conocimiento (Nieves y Haller, 20 I4). Máxime cuando los cambios producidos en el entorno son tan importantes como los que se han dado en la intermediación turística. En este trabajo nos planteamos estudiar cómo Amadeus, uno de los agentes más representativos del desarrollo tecnológico en este sector (Borja Solé y Gomis López, 2009; Sismanidou et al., 2009), se está enfrentando a sus cambios estructurales.

\section{Una aproximación al concepto modelo de negocio desde el enfoque de Recursos y Capacidades}

En el ámbito de la Dirección Estratégica de la empresa, el modelo de negocio puede definirse como el conjunto de decisiones que toma el empresario y las consecuencias derivadas de ellas (Casadesus-Masanell y Ricart, 2007); en este sentido, las decisiones centrales girarán en torno a la oferta de bienes y servicios, los recursos necesarios y la forma de emplearlos, así como el soporte legal de las transacciones realizadas a lo largo del proceso. Por ello permite, de forma sencilla e intuitiva, conocer tanto el modo en que una empresa trabaja y es capaz de alcanzar sus objetivos (Magretta, 2002), como las consecuencias directas e indirectas que provocan los cambios procedentes del entorno o surgidos desde la propia compañía.

Ante los actuales entornos complejos y dinámicos, se considera que el éxito empresarial está, en gran medida, detrás del empleo de este instrumento (Vives y Svejenova, 2009), por lo que debe ser un elemento básico en todo proceso de Dirección Estratégica (Chesbrough y Rosenbloom, 2002; Ricart,
2009), que aún requiere de una mayor profundidad teórica en ciertos campos de estudio (Teece, 2010 ).

El modelo de negocio surge como un nuevo esquema de trabajo en el campo de la Dirección Estratégica con un importante peso en la literatura y que ha sido abordado desde diferentes perspectivas. El Enfoque de Recursos y Capacidades (Wernerfelt, 1984; Barney, 1991; Grant, 1991) resulta interesante a la hora de profundizar en el estudio del funcionamiento del modelo de negocio de una organización. En este sentido permite, por un lado, describir el funcionamiento de la empresa en términos de sus recursos y capacidades esenciales y, por otro lado, identificar, comprender y localizar las fuentes de las ventajas competitivas sostenibles y apropiables sobre las que descansa la creación de valor. Pero además, el Enfoque de Recursos y Capacidades puede verse notablemente enriquecido si se incorpora el modelo de negocio como herramienta, puesto que permite tener una visión holística de la empresa, contribuyendo a conocer mejor cómo funciona la organización en su conjunto, cómo los recursos y competencias esenciales son empleados a lo largo de toda la cadena de valor y, en caso de ser necesario, cómo la empresa redefine su funcionamiento -y posiblemente su gama de recursos y capacidades- en respuesta a un cambio en las condiciones competitivas.

Sin embargo los autores no coinciden en una conceptuación común que facilite el avance en su investigación (Zott, Amit y Mass, 20l I). El problema sobre esta falta de convergencia en la definición lo encontramos en el hecho de que se ha utilizado para definir realidades muy distintas -negocio electrónico, el fenómeno low cost, cambios en la distribución (Alamdari y Mason, 2006; Malighetti, Paleari y Redondi, 2009; Tretheway y Markhvida, 2014)- y a través de corrientes de investigación diversas - gestión de la innovación, gestión de la tecnología, gestión de recursos y capacidades, estrategia, etc., (Rajala y Westerlund, 2007; Zott y Amit, 2008; Ricart, 2009; Al-Debei y Avison, 20 I0)-. No obstante, puede encontrase un punto de conexión en todas las corrientes, al considerar que con esta nueva herramienta de trabajo, en primer lugar, se superaría las fronteras de la empresa para analizar su papel en las redes donde interactúa y, en segundo lugar, se incluiría la arquitectura relacionada con la creación y los mecanismos de apropiación del valor obtenido (Rajala y Westerlund, 2007; Zott y Amit, 2008; Teece, 2010 ; Zott et al., 20|1). 
En definitiva, a partir de las diferentes aproximaciones teóricas al concepto de modelo de negocio, se puede concluir la existencia de tres factores o variables clave sobre los que la dirección debe reflexionar -bien como causa o bien como consecuencia deseable- a la hora de diseñar su estrategia empresarial (Amit y Zott, 200I): el contenido, la estructura y el gobierno.

Desde una perspectiva fundamentalmente estática, el contenido se refiere tanto al conjunto de recursos organizativos con que cuentan las empresas para desarrollar su actividad como a los productos finales que se obtienen para satisfacer las demandas del mercado. Por tanto esta primera variable se desagrega, a su vez, en dos dimensiones, input u output, según se trate, bien de los recursos empleados, bien de los productos finales obtenidos (Amit y Zott, 200 I; Svejenova, Planellas y Vives, 2008; Demil y Lecocq, 2009).

Cabe incorporar en esta primera dimensión el concepto denominado por distintos autores proposición de valor, en clara referencia a lo que la empresa ofrece a los mercados y le permite situarse en una posición de ventaja competitiva (Chesbrough y Rosenbloom, 2002; Osterwalder Pigneur y Tucci, 2005; Svejenova et al., 2008; Vives y Svejenova, 2009; Demil y Lecocq, 2009).

En segundo lugar, y adoptando un enfoque de proceso, la variable estructura se corresponde con las capacidades organizativas (Grant, 1991), y alude a la forma de llevar a cabo los procesos de valor, incluyendo aspectos tales como los agentes implicados o la forma en que éstos combinan o intercambian los recursos necesarios. En la mayoría de los modelos esta dimensión se identifica con la cadena de valor de la empresa (Chesbrough, y Rosenbloom, 2002; Osterwalder et al., 2005; Shafer et al., 2005; Vives y Svejenova, 2009). Es por ello que esta dimensión integra las capacidades funcionales de la organización. No obstante, cabría situar también las capacidades dinámicas de la empresa (Teece, Pisano y Shuen, 1997) en el contexto del modelo negocio. Cualquier modificación en las variables del modelo (contenido, estructura o gobierno), orientada a mejorar su posicionamiento, representaría el desarrollo de tales capacidades dinámicas para enfrentarse a las condiciones cambiantes del entorno.

Por último, la variable gobierno alude a los soportes formales o legales en los que se sustentan las relaciones entre los agentes implicados, tales como el diseño de sistemas retributivos a priori o el reparto de beneficios a posteriori. En concordancia con la Teoría de Recursos, la definición de sistemas de gobierno adecuados, permite a la empresa apropiarse del valor creado a partir de las ventajas competitivas obtenidas (Peteraf, 1993). Los autores se han referido a esta tercera dimensión de múltiples formas: potencial de beneficios, margen o modelo de ingresos (Chesbrough y Rosenbloon, 2002; Osterwalder et al., 2005; Demil y Lecocq, 2009), captura de valor (Shafer et al., 2005; Svejenova et al., 2008) o motor de rentabilidad (Vives y Svejenova, 2009).

Ante la elevada obsolescencia y complejidad tecnológica y la alta competitividad de los mercados, las empresas están obligadas a interactuar con otros agentes independientes con quienes mantienen múltiples relaciones (Zott et al., 201 I; Thomas y Wood, 20I4). Por ello, resulta fundamental que el modelo de negocio adoptado permita que la empresa ocupe un lugar destacado en la red de relaciones donde se integra, puesto que reflejará un mayor poder de negociación en los procesos de creación de valor (Lippman y Rumelt, 2003). Este hecho explica que el éxito de un modelo de negocio dependa significativamente de su grado de compatibilidad/complementariedad con los modelos de los agentes con los que interactúa (Chesbrough y Rosenbloom, 2002).

Uno de los errores más habituales a la hora de emplear esta herramienta se desprende de la falta de atención suficiente a las interdependencias entre las tres variables o dimensiones expuestas. En este sentido, Osterwalder et al. (2005) destacan la necesidad de adoptar un enfoque sistémico en el proceso de definición del modelo de negocio. Es decir, atender cuidadosamente a las relaciones existentes y evaluar los efectos que un cambio en cualquiera de las variables puede provocar en el resto del sistema.

En conclusión, y de acuerdo con el Enfoque de Recursos, las dos primeras variables del modelo -contenido y estructura- estarían directamente vinculadas con la creación y sostenimiento de la ventaja competitiva, mientras que los sistemas de gobierno revelarían la habilidad de la compañía para apropiarse las rentas derivadas de una posición favorable en el mercado frente a la competencia, es decir, para capturar el valor creado. La aportación de Shafer et al. (2005), puede ser una de las que hace más explícita dicha relación, al hacer girar su concepción del modelo de negocio en torno a la distinción entre la creación y la captura de valor. 


\section{El modelo de negocio de Amadeus}

Una vez planteado el marco teórico del trabajo, el objetivo de la presente investigación se centra en la descripción y análisis de la compañía Amadeus. Para ello se ha empleado una metodología que se apoya en tres fuentes de información complementarias: I) revisión de la literatura especializada en el sector turístico; 2) entrevistas no estructuradas con expertos del sector y 3) entrevistas en profundidad con directivos de Amadeus España.

En el caso de las fuentes de información primarias, el proceso de obtención de información se llevó a cabo entre octubre de 201 I y marzo de 2012 . Cabe destacar especialmente la realización de entrevistas con directivos de Amadeus España, en concreto con los máximos responsables de las áreas financiera, tecnológica, marketing y recursos humanos, siguiendo una metodología DELPHI.

Para la obtención de la información se realizaron, en una primera ronda, entrevistas en profundidad con cada uno de los responsables para que identificaran los principales factores clave de éxito de la industria, los recursos estratégicos de la compañía, su forma de desplegarlos, la gama de bienes y servicios más relevantes en la actualidad y los de mayor proyección futura, así como los principales agentes externos con los que interactúa y su papel en los procesos de negocio de Amadeus. A partir de las entrevistas realizadas, se elaboraron unos informes preliminares que fueron enviados a todos los directivos con la finalidad de depurar, en una segunda ron$\mathrm{da}$, las opiniones inicialmente planteadas a partir del juicio conjunto de todos los expertos consultados.

De acuerdo con la información recabada, se ha podido establecer el marco de relaciones existentes entre los factores clave que conforman el modelo de negocio de la empresa, con el fin de identificar la filosofía de la organización y las piedras angulares que conforman las fuentes de las presentes y futuras ventajas competitivas sostenibles responsables de la creación de valor.

El modelo descrito a continuación toma como origen el año 2005 y refleja la lógica dominante (Prahalad y Bettis, 1986) que ha orientado la toma de decisiones, de acuerdo con la consecución de los objetivos planificados hasta la actualidad. En el caso de Amadeus, esta lógica gira en torno al desarrollo y explotación de tres capacidades organizativas clave, estrechamente relacionadas entre sí: capacidad de innovación, capacidad de relación con el cliente y capacidad de reconocimiento en el mercado. Estas capacidades conforman la dimensión estructura del modelo de negocio, puesto que son el resultado de la conveniente combinación estructurada de distintos recursos (financieros, humanos y tecnológicos); tales recursos se corresponden con la dimensión contenido, desde un enfoque de input. Finalmente, desde un enfoque de output, la dimensión contenido se completa con la obtención de dos ramas de productos ampliamente consolidados en el mercado turístico: distribución y soluciones tecnológicas.

La alta especificidad y complejidad de las capacidades esenciales sobre las que se sustenta el modelo favorece la creación y sostenimiento de ventajas competitivas, pero exige el establecimiento de un complejo sistema de gobierno capaz de soportar este tipo de transacciones y de apropiarse el valor generado de su explotación; éste se basa en tres mecanismos fundamentales: el desarrollo interno u orgánico, el mercado de compra-venta de empresas y el establecimiento de acuerdos de cooperación a largo plazo.

Los cambios acaecidos en el sector turístico, expuestos anteriormente, han motivado una importante redefinición en la cartera de productos de la empresa entorno a dos grandes líneas de negocio: la distribución a través del desarrollo de su plataforma de venta y el desarrollo de soluciones tecnológicas (figura I).

En cuanto al negocio de la distribución, los especialistas del sector consultados aventuraban la desaparición de los GDS tradicionales. Sin embargo, la empresa ha sabido redefinir este negocio al transformar su sistema de distribución en plataformas de venta con un alto grado de personalización, lo que le ha permitido implantar una clara estrategia de segmentación de mercados. Por un lado, las necesidades de distribución de las agencias viajes, hoteles o compañías de alquiler de vehículos se satisfacen a través de su producto estrella Amadeus Selling Platform. Por otro lado, la especificidad de la demanda de la industria aérea ha sido el origen del desarrollo de una nueva plataforma de gestión, Altea, con un alto componente tecnológico y funcional. Entre estas funcionalidades cabe destacar la automatización de procesos para la gestión integral de clientes de las compañías aéreas, desde la gestión del inventario y los procesos de reserva hasta el control del embarque de viajeros.

Respecto al desarrollo de soluciones tecnológicas, éstas han permitido, por un lado, mejorar la flexibili- 


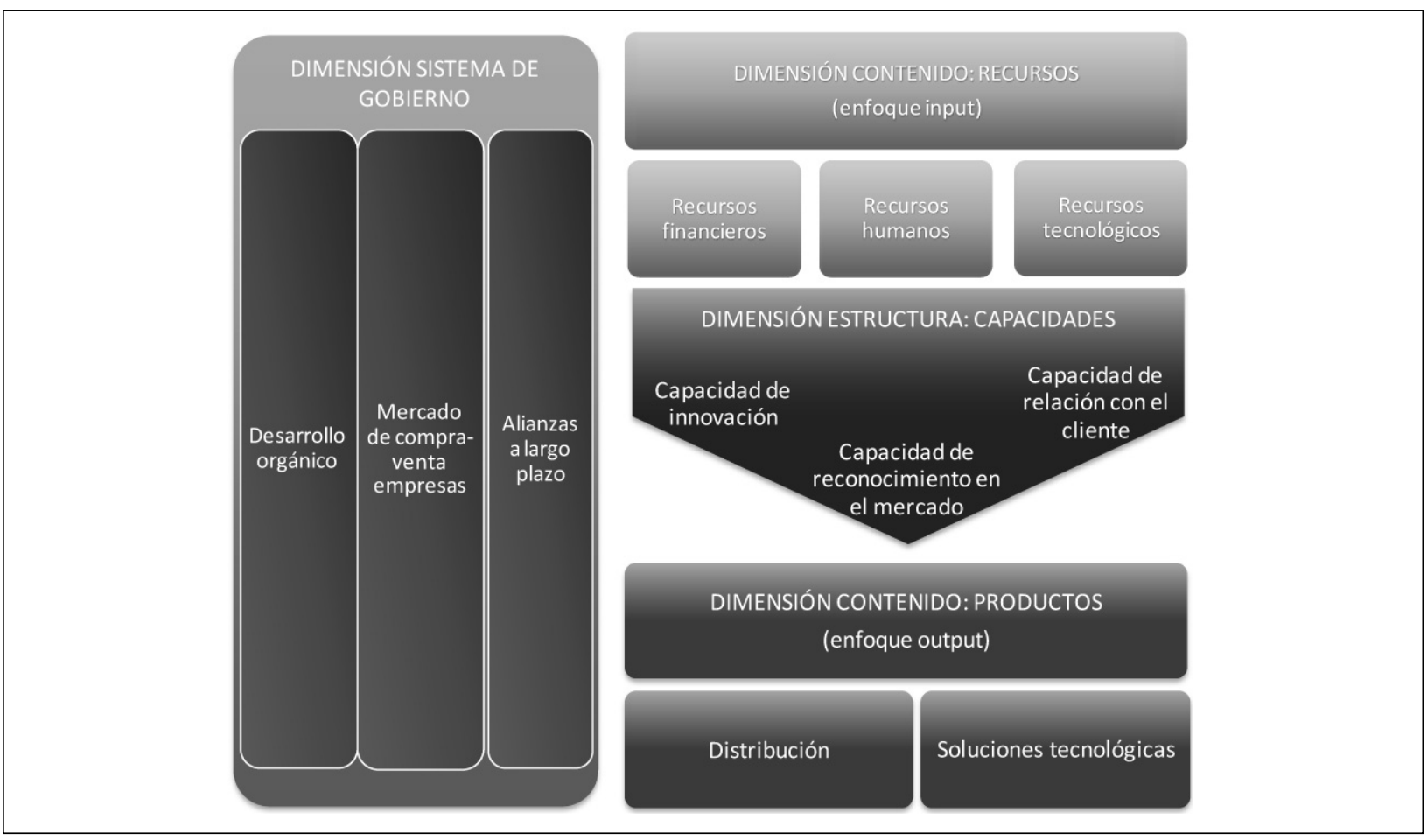

Fuente: Elaboración propia.

Figura I

\section{Esquema conceptual del modelo de negocio de Amadeus}

dad y eficiencia de las plataformas de distribución incorporando nuevos módulos' y, por otro lado, desarrollar otras innovaciones que se incorporan en productos específicos que ofrecen a sus clientes el soporte necesario en todos aquellos espacios que necesiten cubrir y abarcando todas las posibles áreas de desarrollo para sus usuarios ${ }^{2}$. Derivado de todo ello surge el nuevo eslogan de la compañía, «Your technology partnen, reflejando el firme compromiso que Amadeus adquiere con sus clientes.

Esta valiosa cartera de productos es el resultado del compromiso de la empresa con el desarrollo de un complejo y específico sistema de capacidades organizativas sobre la que se asienta en torno a tres pilares básicos: innovación, reputación y relaciones con terceros agentes (figura 2). Dicha estructura del modelo negocio se ha cimentado a lo largo de los años a través de una serie de decisiones fundamentales. En primer lugar, Amadeus desarrolla notables esfuerzos internos para el desarrollo y gestión de sus recursos humanos y tecnológicos. En cuanto a los recursos humanos, la empresa se ha centrado en el desarrollo de actitudes y aptitudes clave entre su personal así como en la implantación de sistemas de gestión de conocimiento que permitan el intercambio de experiencias y buenas prácticas al servicio del modelo de negocio implantado ${ }^{3}$. Respecto al desarrollo de recursos tecnológicos, la compañía ha dedicado una gran parte de su presupuesto a actividades de $1+D+i^{4}$. Este esfuerzo le ha permitido crear una base de conocimiento propio que no solo le ha

\footnotetext{
I Ejemplos de ello se encuentran en módulos relacionados con la venta y el comercio electrónico, tales como Amadeus Business Travel Portal o Amadeus e-Travel Management, que permiten a los clientes corporativos reservar y gestionar sus propios viajes de negocios.

2 En este caso destacan la gestión integral de negocios, a través del back office y herramientas de gestión de clientes, y otros servicios de consultoría, centrados en la formación y el soporte técnico al cliente.

${ }_{3}^{3}$ Un ejemplo es la aplicación on-line Opera Communities and Teamsites que permite compartir archivos, participar en discusiones o trabajar a distancia sobre un mismo documento. Además de flexibilizar el trabajo, esta herramienta ha permitido desarrollar múltiples habilidades sociales como el espíritu colaborativo.

${ }^{4}$ Según el informe de la Comisión Europea «The 2013 EU Industrial R\&D Investment Scoreboard», Amadeus invirtió 440,3 millones de euros en I+D durante 20 I 2, lo que supuso un incremento del 26,7\% con respecto a la inversión realizada en 20 I I. La empresa invierte un $15,1 \%$ de sus ingresos totales en la mejora continua de este concepto lo que le permite liderar las clasificaciones del sector de los viajes y el turismo, tanto a escala europea como internacional.
} 
permitido obtener productos innovadores sino también acometer con éxito la vigilancia tecnológica del entorno que le rodea y, por tanto, ha servido de apoyo a otras decisiones clave, como la evaluación del potencial tecnológico de otras compañías.

En segundo lugar, y apoyándose de forma complementaria en el desarrollo orgánico recién descrito, se ha llevado a cabo un proceso de reorganización del grupo empresarial que ha desembocado en la adquisición de empresas tecnológicamente fuertes -One Rail o Airline Automation Inc., en 2003 u Optims, en 2005-y, desde 201 0, en la venta de empresas que no formaban parte de su core business tecnológico -la agencia de viajes on-line Opodo, el consorcio de Agencia de Viajes Vacation.com o la división de gestión de propiedades de pequeñas cadenas hoteleras
(PMS)-. Estos procesos han permitido dotar de una mayor coherencia a la cartera de negocios, al aprovechar los beneficios derivados de las economías de especialización tecnológica (Rothaermel y Deeds, 2004), y, además, han contribuido tanto al desarrollo de capacidades estratégicas como al saneamiento financiero del grupo.

Otra decisión clave para el desarrollo de las capacidades esenciales del grupo ha sido el establecimiento de acuerdos de colaboración a largo plazo con diversas empresas (por ejemplo IAG Group o Quantas) y el mantenimiento de relaciones estables con clientes, que han permitido orientar los esfuerzos tecnológicos a desarrollar por Amadeus, y crear y mantener una notable reputación ${ }^{5}$ y consolidar una marca de prestigio, que se traduce en una cartera de clien-

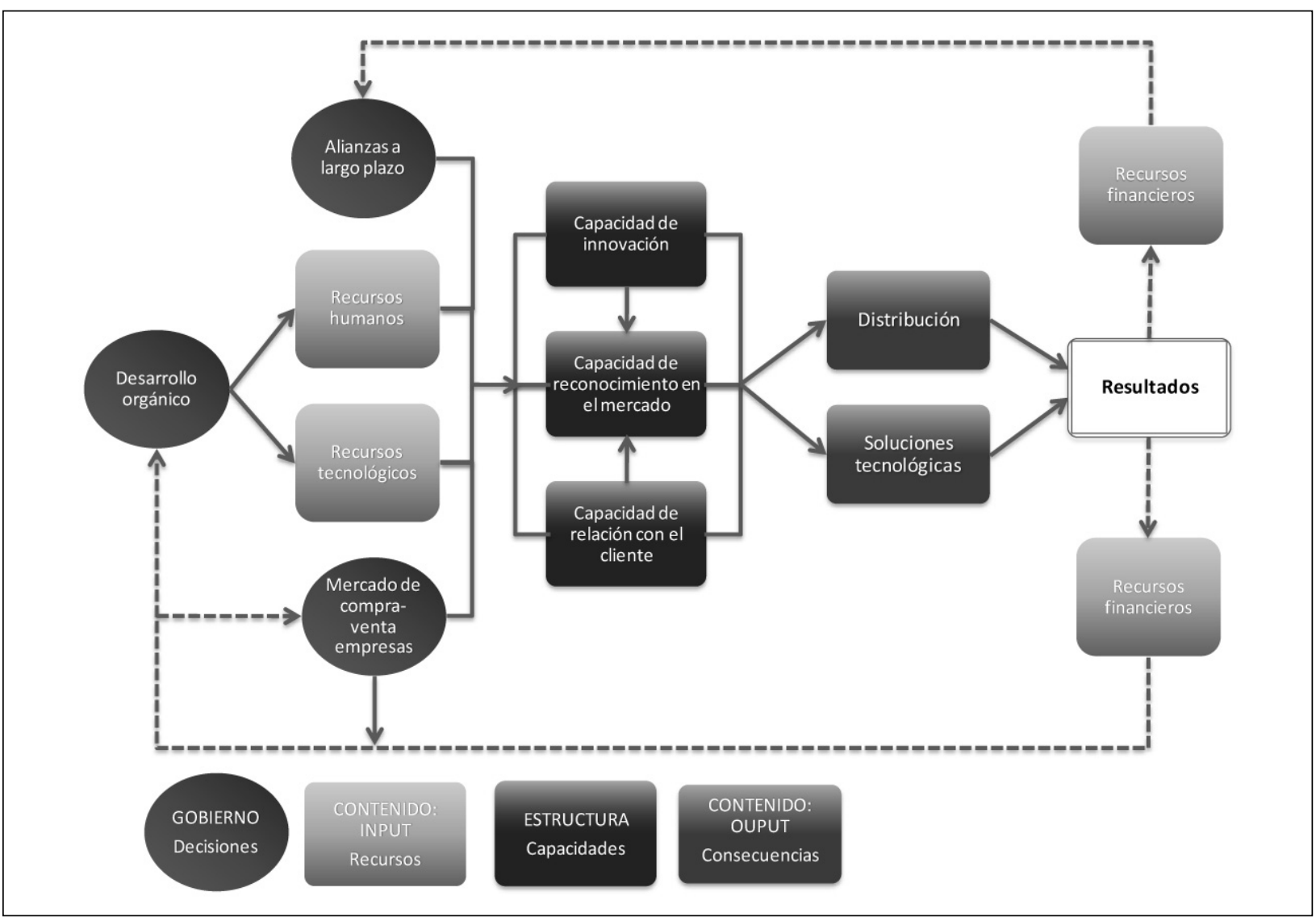

Fuente: Elaboración propia

Figura 2

El modelo de negocio de Amadeus: principales dimensiones

\footnotetext{
${ }_{5}^{5}$ Centrada no solamente en el área económico-financiera de la responsabilidad social, sino también en el área de acción social mediante programas como el MASSIVEGOOD, en el que las soluciones tecnológicas de Amadeus facilitan la donación de pequeñas cantidades de dinero cada vez que se adquiere un billete o se reserva un hotel, o el patrocinio de actividades deportivas como el torneo de Golf $\mathrm{Cl}$ Christel House Opencon, para apoyar el desarrollo de un Modelo Educativo Integral Gratuito en beneficio de niños y jóvenes con pocos recursos a nivel mundial.
} 
tes fieles en ciento noventa y cinco países gracias, en gran medida, al papel de sus 73 filiales comerciales (ACO Amadeus Commercial Organisations), con presencia en 195 países.

\section{Conclusiones: evaluación del modelo de negocio de Amadeus}

En el presente estudio se describe con detenimiento el concepto y estructura del modelo de negocio. Como herramienta estratégica permite comprender de forma clara y sencilla el funcionamiento de una empresa; en primer lugar, hace posible la identificación de los recursos y capacidades clave; en segundo lugar, contribuye al conocimiento de las decisiones que permiten su acumulación y desarrollo y, finalmente, muestran la aportación de dichos recursos y capacidades a los procesos organizativos y, por lo tanto, a la creación de valor.

En consecuencia, ante un cambio en las condiciones del entorno, este instrumento adquiere un especial valor, ya que permite reconocer rápidamente los efectos de dichos cambios y establecer sistemas de adaptación a los mismos. La redefinición de los modelos de negocio supondrá una ventaja competitiva, siempre y cuando en su funcionamiento exista cierta ambigüedad causal (Lippmann y Rumelt, 1982), es decir, cuando las relaciones causa-efecto que se establezcan entre los distintos mecanismos, rutinas o recursos con los resultados conseguidos, no puedan ser comprendidas en su totalidad por las empresas competidoras.

Además del fenómeno anteriormente descrito, el modelo de negocio debe cumplir con las siguientes condiciones para que pueda ser aplicado de forma favorable (Casadesus-Masanell y Ricart, 2007; Demil y Lecocq, 2009; Vives y Svejenova, 2009): I ) refuerzo y virtuosidad, aspectos directamente relacionados con la consistencia interna o sistémica del modelo, 2) robustez, criterio relacionado con su sostenibilidad en el tiempo y 3) rentabilidad, criterio relacionado con los resultados económicos del modelo.

De acuerdo con las reflexiones realizadas, en este estudio se ha planteado una novedosa aplicación de la herramienta al caso de compañía Amadeus, apoyando sus fundamentos en un marco teórico ampliamente asentado en el campo de la Dirección Estratégica como es la Teoría de Recursos y Capacidades.
De esta forma, se muestra cuáles son las principales variables sobre las que descansan sus resultados y su actual posicionamiento competitivo.

Cabe considerar que el modelo de negocio de Amadeus sea virtuoso, entre otras razones, por la complementariedad existente entre las diferentes capacidades que conforman la dimensión estructura; en este sentido, la estrecha relación con los clientes orienta los esfuerzos de innovación que deben realizarse y ambas capacidades redundan en la mejora de la reputación de la empresa. Otro ejemplo se encuentra en el apoyo que proporciona el desarrollo de las actividades internas tecnológicas a los procesos de compra-venta de empresas, al dotar a la compañía de los conocimientos necesarios para evaluar el potencial de otras organizaciones. Por otra parte, el modelo puede considerarse robusto como consecuencia de la complejidad y especificidad de las capacidades, que dificultan su imitación, sustitución y transferencia y, por tanto, contribuyen al sostenimiento de la ventaja competitiva. Tampoco debe olvidarse que el desarrollo de las capacidades que le permiten disfrutar de una posición de ventaja en el mercado, facilita la negociación con terceros agentes y el establecimiento de sistemas de gobierno favorables que promuevan la apropiación de valor económico creado. Por ello, empleando la terminología propia de la literatura sobre modelos de negocio, podemos considerar que estas capacidades conforman las consecuencias rígidas, derivadas de las decisiones clave por parte de la Dirección, de muy difícil replicación por otras empresas de la industria.

Además, el conocimiento que incorporan dichas capacidades le otorga la flexibilidad necesaria para adaptarse al dinamismo del entorno a través de la constante renovación/ampliación de su cartera de productos; en consecuencia, estos productos constituyen las consecuencias flexibles -objetivos a corto plazo- que se traducen en recursos financieros inmediatos.

El análisis de la información financiera permite hacer una valoración global de la idoneidad del modelo de negocio de Amadeus. En este sentido, los resultados del ejercicio anual de 2013 registraron unos ingresos de 3.103,7 millones de euros y un EBITDA de I.। 88,7 millones de euros ${ }^{6}$, poniendo de manifiesto la fortaleza económica del modelo de negocio, ya que en los últimos años el crecimiento de ambas cifras se ha consolidado. A pesar de todo lo anterior-

${ }^{6}$ Dato suministrado por Amadeus a la Comisión Nacional del Mercado de Valores (CNMV), informe 2013. 
mente comentado, en la actualidad debe reconocerse que Amadeus tiene un desafío de cara al futuro, mejorar su potencial para apropiarse las rentas generadas. En este sentido, el margen sobre los beneficios aunque positivo en 2013 tras la caída en 2012, aún es mejorable; por ello, a través de la estructura del modelo de negocio, se deberá identificar las principales causas de este problema y abordar las decisiones que se consideren oportunas.

En definitiva, la descripción de un modelo de negocio como el aquí presentado es de gran utilidad para la Dirección de cualquier empresa; en este sentido, permite evaluar de forma sistemática el ajuste del modelo con el entorno que le rodea, así como la coherencia de cualquier decisión con la lógica dominante de la compañía.

\section{BIBLIOGRAFÍA}

ALAMDARI, F., and MASON, K. (2006): «The future of airline distribution». Journal of Air Transport Management, $12,122-134$.

AL-DEBEI, M.M., and AVISON, D. (20I0). «Developing a unified framework of the business model concept». European Journal of Information Systems, 19, pp. 359-376.

AMIT, R., and ZOTT, Ch. (200I). «Value creation in e-business». Strategic Management Journal, 22, pp. 493-520.

BARNEY, J. (1991). «Firm resources and sustained competitive advantage». Journal of Management, 17 (I), pp. 99- 120.

BORJA SOLÉ, L., y GOMIS J. M. (2009). El nuevo paradigma de la intermediación turística, Madrid: Ed. Pirámide.

BRADLEY, K. ( 1997). «Intellectual capital and the new wealth of nations». Business Strategy Review, 8 ( I), pp. 5362.

BUHALIS, D., Y LICATA, M.C. (2002): «The future of eTourism intermediaries». Tourism Management, 23 (3), pp. 207- 220.

BUHALIS, D., y LAW, R. (2008). «Progress in information technology and tourism management: 20 years on and 10 years after the internet e the state of etourism research». Tourism Management, 29 (4), pp. 609-623.

CALERO GARCÍA, F., PARRA LÓPEZ, E., y SANTANATALAVERA, A. (20 I 0). «Vigilancia tecnológica e inteligencia competitive: Un análisis de la demanda tecnológica en alojamientos turísticos en Canarias». Revista de Análisis Turísticos, 9, pp. 30-4I.

CASADESUS-MASANELL, R., and RICART, J.E. (2007). «Competing through business models», Working paper IESE Business School, 7I 3. Universidad de Navarra.
CHESBROUGH, H., and ROSENBLOOM, R.S. (2002). «The role of business model in capturing value from innovation: Evidence from Xerox corporation's technology spin-off companies». Industrial and Corporate Change, I I, pp. 529-555.

DEMIL, B., y LECOCQ, X. (2009). «Evolución de modelos de negocio. Hacia una visión de la estrategia en términos de coherencia dinámica». Universia Business Review, 23, pp. 86- 107.

FLORES ALBERTO, D., SALAZAR NIEBLA, L., y SANTANATURÉGANO, M.A. (20I I). «¿Desaparecerán losTour Operadores? El papel de los intermediarios en la distribución turística: Análisis del caso de Tenerife». PASOS. Revista de Turismo y Patrimonio Cultural, 9 (2), pp. 34I35 I.

GRANADOS, N., KAUFFMAN, R., y KING, B. (2008): «How has electronic travel distribution been transformed? A test of the theory of newly vulnerable markets». Journal of Management Information Systems, 25 (2), 73-96.

GRANT, R.M. (199|). «The Resource-Based Theory of competitive advantage: Implications for strategy formulation». California Management Review, 34, primavera, pp. ||4-|35.

HO, C.I., and LEE,Y.L. (2007). «The development of an etravel service quality scale». Tourism Management, 28 (6), pp. | 434- 1449.

$\mathrm{KOCH}, \mathrm{M}$., and MCGRATH, R. (1996). «lmproving labor productivity: Human resource management policies do matter». Strategic Management Journal, I7 (5), pp. 335 354.

LIPPMAN, S. A., and RUMELT, R. P. (1982). «Uncertain Imitability: An Analysis of interfirm differences in efficiency under competition». The Bell Journal of Economics, 13 (2), pp. 4 I8-438.

LIPPMAN, S.A., and RUMELT, R.P. (2003). «A bargaining perspective on resource advantage». Strategic Management Journal, 24, pp. $1069-1086$.

LLACH, J., MARIMON, F., ALONSO-ALMEIDA, M., and BERNARDO, M. (20|3). «Determinants of online booking loyalties for the purchasing of airline tickets». Tourism Management, 35, pp. 23-31.

MAGRETTA, J. (2002). «Why business model matter?». Harvard Business Review, mayo, pp. 86-92.

MALIGHETTI, P., PALEARI S., and REDONDI, R. (2009). «Pricing strategies of low-cost airlines:The Ryanair case study». Journal of Air Transport Management, I5, pp. 195 203.

NIEVES, J., HALLER, S. (20 I 4). «Building dynamic capabilities through knowledge resources». Tourism Management, 40, pp. 224-232. 
OSTERWALDER, A., PIGNEUR, Y., and TUCCI, C. (2005). «Clarifying business models: Origins, present, and future of the concept». Communications of the Association for Information Systems, I5, pp. 5 I-775.

PETERAF, M.A. (1993). «The cornerstones of competitive advantage: A Resource-Based View». Strategic Management Journal, |4, pp. |79-191.

PORTER, M. (200I). «Strategy and the Internet». Harvard Business Review, 103D, pp. 63-78.

PRAHALAD, C.K., and BETTIS, R.A. (1986). «The Dominant Logic: A new linkage between diversity and performance». Strategic Management Journal, 7, pp. 485-50I.

RADULOVI_,L. (20 I 3). «The role and potential of Global Distribution System Amadeus forTourism development at the global level». Singidunum Journal, 10 (I), pp. 2838.

RAJALA, R., and WESTERLUND, M. (2007). «Business models - a new perspective on firms' assets and capabilities». Entrepreneurship and innovation, 8 (2), I I 5- 125.

RICART, J.E. (2009). «Modelo de negocio: El eslabón perdido en la Dirección Estratégica». Universia Business Review, 23, pp. 12-25.

ROTHAERMEL, F.T., and DEEDS, D.L. (2004). «Exploration and exploitation alliances in biotechnology: A system of new product development». Strategic Management Journal, 25, pp. 20l-221.

SHAFER, S.M., SMITH, H.J., and LINDER, J.C. (2005). «The power of business models». Business Horizons, 48, pp. 199-207.

SISMANIDOU, A., PALACIOS, M., and TAFUR, M. (2009). «Progress in airline distribution systems: The threat of new entrants to incumbent players». Journal of Industrial Engineering and Management, 2 ( I), pp. 25 I-272.

SPENCER, A.J., BUHALIS, D., and MOITAL, M. (20I2). «A hierarchical model of technology adoption for small owner-managed travel firms: An organizational decision- making and leadership perspective». Tourism Management, 33, pp. I195- 1208.

SVEJENOVA, S., PLANELLAS, M., andVIVES, L. (2008). «Business model in motion: Insights from the case of Ferran Adrià». Business Model Conference. City University, Londres.

TEECE, D.J., PISANO, G., and SHUEN, A. (1997). «Dynamic capabilities and strategic management». Strategic Management Journal, 18, pp. 509-533.

TEECE, D.J. (20 I0). «Business Models, Business Strategy and Innovation». Long Range Planning, 43 (2-3), pp. I72194.

The 2013 EU Industrial R\&D Investment Scoreboard (20।3). European Commission-Joint Research Centre. En: http://iri.jrc.ec.europa.eu/scoreboard I 3.html

THOMAS, R., and WOOD, E. (20|4). «Innovation in tourism: Re-conceptualising and measuring the absorptive capacity of the hotel sector». Tourism Management, 45, pp. 39-48.

TRETHEWAY, M.W., and MARKHVIDA, K. (20I4). «The aviation value chain: Economic returns and policy issues». Journal of Air Transport Management, 4I, pp. 3-16

TSOUKAS, H. (2005). «Complex knowledge, studies in organizational epistemology». Oxford University Press, Nueva York.

VIVES, L., y SVEJENOVA, S. (2009). «Innovando en el modelo de negocio. La creación de la Banca Cívica». Universia Business Review, 23, pp. 70-85.

WERNERFELT, B. (1984). «A resource-based view of the firm». Strategic Management Journal, 5 (2), pp. 17| - 80.

ZOTT, CH., and AMIT, R. (2008). «The fit between product market strategy and business model: Implications for firm performance». Strategic Management Journal, 29, pp. I-26.

ZOTT, CH., AMITT, R., and MASSA, L. (201 I). «The business model: Recent developments and future research». Journal of Management, 37 (4), pp. I019-1042. 
WWW.revistadyo.com

\title{
La importancia de los recursos humanos en la cadena de suministro The importance of human resources in the supply chain
}

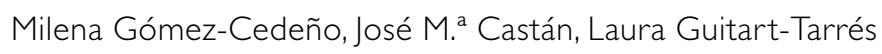

Universidad de Barcelona. Facultad de Economía y Empresa. Departamento de Economía y Organización de Empresas. milgomez@ub.edu,jmcastan@ub.edu, laura.guitart@ub.edu

Fecha de recepción: 16-5-2014

Fecha de aceptación: 24-7-2014

Resumen: La Gestión de los Recursos Humanos (HRM) con un enfoque en la Cadena de Suministro (SC) permite a las empresas gestionar eficazmente sus cadenas de suministros. Este artículo justifica la importancia del estudio de los Recursos Humanos en la Cadena de Suministro (HRSC) y aporta un análisis de las investigaciones en estos dos campos, que permite aflorar las potencialidades y carencias sobre su estudio. Se presenta el estado del arte sobre la HRSC a través de un análisis bibliométrico de las investigaciones académicas publicadas en las últimas décadas, con el objetivo de identificar la existencia de vacíos en la literatura. Además, analizan los métodos de investigación utilizados, las áreas temáticas abordadas y las principales contribuciones de estas investigaciones. Como resultado, se han localizado 46 publicaciones que destacan el gran potencial de la HRSC, a pesar que existe una gran carencia de estudios sobre esta temática y se evidencia que es un área olvidada en la investigación, el interés por la cual surgió hace tan sólo dieciséis años. El estado del arte constituye el marco teórico adecuado para comprobaciones empíricas posteriores, lo cual implica el establecimiento y la justificación de nuevas líneas de investigación. A pesar de demostrar la importancia de los recursos humanos $(\mathrm{RRHH})$ en la mejora de la SC, no existe hasta el momento un análisis bibliométrico como el que se presenta en este trabajo.

Palabras Claves: Gestión de los Recursos Humanos (HRM), Gestión de la Cadena de Suministro (SCM), Estudio bibliométrico.

Abstract: The interest of this study is based on the fact that is no similar studies done in the past, despite being shown the great potential of the study of Human Resources in the Supply Chain (HRSC), which allowed companies to effectively manage their supply chains. Therefore, this work is a novel of great interest to academics and practitioners in the management of the supply chain. The fields of study of Human Resources Management (HRM) and Supply Chain Management (SCM), historically have been treated separately, although they are "intimately linked" in most business environments. The Human Resources Management (HRM) with a focus on the Supply Chain (SC) enables companies to effectively manage their supply chains. This paper justifies the importance of the study of human resources in the supply chain (HRSC) and provides an analysis of the research in these two fields, which allows the potential and shortcomings emerge on their study. The state of the art on the human resources in the supply chain (HRSC) is presented through a bibliometric analysis of published academic research in recent decades, with the aim of identifying the existence of gaps in the literature. In addition, analyze the research methods used, the thematic areas addressed and major contributions of this research. As a result, we have located 46 publications that highlight the great potential of the human resources in the supply chain (HRSC), eventhough there is a lack of studies on this subject and it is evident that this is a neglected research area; with an interest which arose only sixteen years ago. The management of human resources to improve Supply Chain Management (SCM) practices has not yet been treated formally, although some research suggests that this effort would improve the performance of the Supply Chain Management (SCM). The state of the art is the appropriate theoretical framework for further empirical tests, which implies the establishment and justification of new lines of future research. We show that future research should be oriented towards the study of soft or non-structural components, with the intention of overcoming some of the critical factors in the supply chain, such as human resources.

Keywords: Human Resources Management (HRM), Human Capital, Supply Chain Management (SCM), Bibliometric study.

\section{Introducción}

La Gestión de la Cadena de Suministro (SCM) es la integración de los procesos clave de negocio en el suministro de productos, servicios e información, que añaden valor, desde los proveedores hasta el usuario final. En este sentido, la SCM tiene una importante dimensión humana debido a su énfasis en la comunicación y la cooperación entre todas las partes que comprenden la cadena de suministro (SC).
Tracey (2007) considera que la SCM debe ser un enfoque de negocio basado en la cooperación, no sólo a través de todas las áreas funcionales de la compañía, sino también a través de los miembros de la propia cadena. Así, este enfoque basado en la colaboración es lo que da a la SCM su dimensión humana.

Contradictoriamente y según la literatura existente, los trabajos sobre SCM han sido dirigidos mayorita- 
riamente al estudio de las áreas estructurales o hard ', más no así al de las denominadas áreas soft o no estructurales $^{2}$ (Giunipero et al., 2008; Shub et al., 2009; Fisher et al., 20l0), por lo que existen lagunas que ocupar dentro de la literatura. Si bien en un principio las empresas centraban su atención en las decisiones estructurales, posteriormente se observó la importancia de las no estructurales. Es más, el impacto acumulativo a largo plazo de las decisiones no estructurales o soft puede ser tan importante como el de las estructurales o hard (Fernández et al., 2003).

Así, el estudio de estos aspectos más suaves de la SC, al estar la cadena de suministros inmersa en una compleja red de relaciones, se ha convertido en un gran desafío para las empresas. Pese a los avances y aportaciones sobre el estudio de los aspectos técnicos, existen autores que consideran que quizás sean las personas quienes dificultan el funcionamiento de la SC (Fawcett et al., 2008).

En este sentido, con el propósito de dilucidar las potencialidades y las carencias existentes en el estudio de los Recursos Humanos en la Cadena de Suministro (HRSC), se considera oportuno reflexionar sobre aspectos tales como: (I) ¿qué áreas temáticas han sido analizadas en las investigaciones sobre SCM?; (2) iexisten lagunas dentro de la literatura?; (3) ies el estudio de los RRHH una de estas lagunas? (4) ¿cuáles son los beneficios, barreras y puentes para el éxito de la SC?;
(5) ¿hacia dónde deberían dirigirse las investigaciones futuras?

La estructura de este trabajo es la siguiente. En primer lugar, se realiza un análisis de las publicaciones sobre SC, con el fin de identificar la existencia de vacíos dentro de la literatura. En segundo lugar, se realiza un análisis comparativo entre las diferentes áreas temáticas abordadas, para dilucidar el potencial de los HRSC. En tercer lugar, debido a la ausencia de trabajos en este campo, se hace un análisis bibliométrico sobre la HRSC (frecuencia de publicación, metodologías utilizadas y clasificación por categorías temáticas) para, por último, realizar un análisis de contenido de las investigaciones identificadas, analizando las contribuciones aportadas por los investigadores en estas dos disciplinas (RRHH y SC). Finalmente, se establecen algunas sugerencias dirigidas a orientar futuras investigaciones.

\section{Los estudios sobre la cadena de sumininistro y la gestion de recursos humanos}

En estudios previos de algunos investigadores, como es el caso de Giunipero et al. (2008) ${ }^{3}$, se clasifican las investigaciones sobre SC publicadas en un período de 10 años (1996-2006) en las categorías temáticas que recoge la Tabla 1 .

Tabla I

Categorías temáticas de las investigaciones sobre SC

\begin{tabular}{|c|c|c|c|}
\hline \multicolumn{2}{|r|}{ Categoría Temática } & \multirow{2}{*}{$\frac{\text { Total }}{95}$} & \multirow{2}{*}{$\begin{array}{c}\text { Porcentaje } \\
23 \%\end{array}$} \\
\hline I & Estrategia SCM & & \\
\hline 2 & Marco, tendencias y desafíos & 74 & $18 \%$ \\
\hline 3 & Alianzas & 66 & $16 \%$ \\
\hline 4 & E-commerce & 32 & $8 \%$ \\
\hline 5 & Estrategias-tiempo & 26 & $6 \%$ \\
\hline 6 & Calidad & 22 & $5 \%$ \\
\hline 7 & Tecnologías de la información (IT) & 20 & $5 \%$ \\
\hline 8 & Desarrollo/selección y gestión de proveedores & 16 & $4 \%$ \\
\hline 9 & Outsourcing & 13 & $3 \%$ \\
\hline 10 & Medioambiente/Responsabilidad social & 12 & $3 \%$ \\
\hline । & SMC global & 12 & $3 \%$ \\
\hline 12 & Comportamiento del consumidor & 9 & $2 \%$ \\
\hline 13 & Gestión de Recursos Humanos & 8 & $2 \%$ \\
\hline & Total & 405 & $100 \%$ \\
\hline
\end{tabular}

Fuente: Adaptado de Giunipero et al. (2008) a partir de la clasificación del Institute for Supply Chain Management

\footnotetext{
I Áreas estructurales o hard se refieren a la tecnología, uso de la información o sistemas de medición, JIT, IT, compras, entre otros.

2 Áreas soft o no estructurales guardan relación con los recursos humanos (RRHH), la cultura organizacional, la confianza, entre otros.

3 Este autor analiza el comportamiento de las investigaciones sobre SC, a través de una revisión de la literatura basada en la clasificación de temas que propone el Institute for Supply Chain Management (ISM).
} 
Los resultados obtenidos por Giunipero et al. (2008) muestran como las investigaciones sobre SC se han decantado hacia algunas categorías (como por ejemplo, la estrategia en SCM), evidenciando ausencias en otras temáticas. Éste es el caso del área de la Gestión de los Recursos Humanos, definida como «el proceso de establecer las relaciones jerárquicas necesarias, así como los aspectos de los recursos humanos (RRHH) que afectan al rendimiento diario del personal de la SC (cambio organizacional, organizaciones virtuales, eficacia organizacional, capacidad de respuesta frente a la gestión de los estilos de anticipación, habilidades de aprendizaje organizacional, conocimiento tácito, funciones laborales, conflictos de roles y habilidades de compra) » ${ }^{4}$, la cual tan sólo ha sido estudiada en un $2 \%$ de las investigaciones sobre SC.

Como plantea Scarbrough (2000), «la SC genera una demanda importante en la gestión de los recursos humanos para la obtención de las habilidades y la flexibilidad de los comportamientos necesarios para alcanzar una integración más estrecha». Por otro lado, según Tracey (2007), la sincronización de la SC depende de las personas involucradas puesto que éstas están presentes en los procesos y en la tecnología utilizada. Además, la ventaja competitiva no se logra únicamente a través de la inversión en tecnología de la información ya que existen muchas situaciones que requieren la aplicación del juicio humano. En este sentido, también Tracey (2007) señala que un principio fundamental de la SCM es mejorar la eficacia de los participantes individuales.

A pesar de ello, son pocas las investigaciones que han analizado la relación entre la HRM y el rendimiento de la SCM (Giunipero et al., 2008; Shub, 2009; Fisher et al., 20 I0). La evidencia indica que las investigaciones sobre SCM han sido orientadas al estudio de la integración de los procesos de fabricación y comercialización, centrándose en la medición del desempeño de las prácticas de SCM sobre todo en aspectos operativos, tales como la eficiencia de recursos y reducción de costes, y aspectos de marketing, tales como servicio al cliente. Sin embargo, la HRM para la mejora de las prácticas de SCM aún no ha sido tratada de manera formal, a pesar de que algunas investigaciones indican que este esfuerzo mejoraría el rendimiento de la SCM (Gowen, 2003).

En esta dirección, el estudio efectuado por Fawcett et al. (2008) sobre los diez beneficios, barreras y puentes/oportunidades para lograr el éxito de la $\mathrm{SCM}^{5}$ (recogidos en la Tabla 2 ) sugiere que $\ll \ldots$ la

Tabla 2

Beneficios, barreras y oportunidades para la gestión estratégica de la SC

\begin{tabular}{|c|c|c|c|}
\hline & Beneficios & Barreras & Puentes/Oportunidades \\
\hline & Enfoque hacia el cliente & Rivalidad Intra-empresas & Operaciones, procesos y SCM \\
\hline । & Aumento de la capacidad de respuesta & Inadecuado intercambio de información & Precisión en los objetivos globales \\
\hline 2 & Incremento de sensibilidad con el cliente & Objetivos operativos inconsistentes & $\begin{array}{c}\text { Alineación y estandarización del } \\
\text { proveedor }\end{array}$ \\
\hline 3 & Satisfacción al cliente & $\begin{array}{l}\text { La falta de disposición para compartir } \\
\text { riesgos y beneficios. }\end{array}$ & El uso eficaz de los proyectos pilotos. \\
\hline 4 & $\begin{array}{l}\text { Cumplimiento en las entrega en } \\
\text { tiempos más cortos. }\end{array}$ & $\begin{array}{l}\text { La falta de disposición para compartir } \\
\text { información. }\end{array}$ & $\begin{array}{c}\text { Prioridad de documentación en el } \\
\text { proceso de información }\end{array}$ \\
\hline & Empresa & Complejidad Gerencial & Gestión de personas \\
\hline 5 & Reducción de los costes de compra & La falta de alianza en las directrices. & Directivos y personal de apoyo \\
\hline 6 & Mejor utilización de activos & $\begin{array}{l}\text { Procesos mal valorados en relación a los } \\
\text { costes }\end{array}$ & Abrir el intercambio de información \\
\hline 7 & $\begin{array}{l}\text { Mejor capacidad de Respuesta ante } \\
\text { situaciones inesperadas }\end{array}$ & Medidas No Alineadas & Alianzas basadas en la confianza \\
\hline 8 & Reducción de los costes de inventario & Los límites de la organización & $\begin{array}{c}\text { Directivos capacitados y con experiencia } \\
\text { multifuncional. }\end{array}$ \\
\hline 9 & Productividad en la empresa & Medición de la contribución de SC. & Educación y formación sobre la SC. \\
\hline 10 & Reducción del coste total del producto & Medición de la demanda del cliente & $\begin{array}{c}\text { Aprovechamiento de los consejos } \\
\text { directivos de la SC }\end{array}$ \\
\hline
\end{tabular}

Fuente: Fawcett et al. (2008)

\footnotetext{
${ }^{4}$ Definición de la categoría "HRM" según el estudio de la SC del Institute for Supply Chain Management (ISM).

${ }^{5}$ A través de un análisis cuantitativo y cualitativo, mediante encuestas por correo y el análisis de 5 I casos en profundidad.
} 
SCM está habilitada por la tecnología moderna de la información, pero el éxito de la SCM se basa en las personas». En realidad «las personas son el puente o la barrera para la colaboración en la SC».

A partir de la información de la Tabla 2, se observa que las principales barreras se encuentran en los aspectos estructurales o hard (tecnología, información y sistemas de medición, entre otros), mientras que los problemas de las personas son más difíciles de solventar (la ausencia de confianza, la aversión al cambio, la falta de compromiso, culturas incompatibles y las malas prácticas de $R R H H$ ). Sin embargo, las personas podrían ser la clave para el éxito de la SCM y deben ser tomadas en cuenta con el mismo énfasis con el que son atendidas las principales barreras.

En consecuencia, tal como se establece en la literatura, la HRM permite a las empresas gestionar eficazmente sus cadenas de suministros. Así, en este trabajo se plantea la siguiente pregunta de investigación: ¿cómo ha sido abordado el estudio de los $\mathrm{RRHH}$ bajo el enfoque de SC en la literatura de SCM?

\subsection{El potencial de los estudios de Recursos Humanos relacionados con la SCM}

A pesar de las potencialidades de la gestión conjunta de la SC y los RRHH para que las empresas administren eficaz y eficientemente sus SC, se ha presta- do poca atención a su estudio (Giunipero et al., 2008; Fawcett, 2008; Shub et al., 2009; Fisher et al., 2010 ; Gómez et al., 20।3a; Gómez et al., 2013b).

Esta idea se demuestra en la tabla 3, el cual se ha construido a partir de un eje vertical que contiene las 13 categorías temáticas establecidas por el Institut of Supply Chain Management (ISM). Para estas trece categorías se visualizan las siguientes tres dimensiones: ( I) Publicaciones: Resultados de la revisión de la literatura realizada por Giuinipero et al. (2008) sobre las publicaciones en las 13 categorías de SC del ISM en los diez años del estudio. (2) Barreras: Las barreras establecidas por Fawcett et al. (2008) (agrupadas dentro de las 13 categorías temáticas de la clasificación establecida por el ISM). (3) Puentes: Los puentes u oportunidades para alcanzar el éxito, propuestos por Fawcett et al. (2008) (agrupadas dentro de las 13 categorías temáticas de la clasificación establecida por el ISM).

En esta misma tabla, el eje horizontal contiene el porcentaje de estas tres dimensiones dentro de cada una de las 13 categorías temáticas. Así, por ejemplo, en relación a la estrategia en SCM, el 23\% de las publicaciones de la revisión de la literatura de Giunipero et al. (2008), el 30\% de las barreras establecidas por Fawcett et al. (2008) y el $20 \%$ de los puentes establecidos por Fawcett et al. (2008) se refieren a esta categoría.

Tabla 3

Temas estudiados sobre SCM versus las 10 principales barreras y puentes para el éxito de la SCM

\begin{tabular}{|c|c|c|c|c|}
\hline & Categoría & Publicaciones & Barreras & Puentes/oportunidades \\
\hline । & Estrategia en SCM & $23 \%$ & $30 \%$ & $20 \%$ \\
\hline 2 & Marcos, Tendencias y desafíos & $18 \%$ & $0 \%$ & $0 \%$ \\
\hline 3 & Alianzas & $16 \%$ & $30 \%$ & $0 \%$ \\
\hline 4 & E-commerce & $8 \%$ & $10 \%$ & $0 \%$ \\
\hline 5 & Estrategias-tiempo & $6 \%$ & $20 \%$ & $0 \%$ \\
\hline 6 & Calidad & $5 \%$ & $10 \%$ & $0 \%$ \\
\hline 7 & IT & $5 \%$ & $0 \%$ & $10 \%$ \\
\hline 8 & Desarrollo/selección y gestión de proveedores & $4 \%$ & $0 \%$ & $10 \%$ \\
\hline 9 & Outsourcing & $3 \%$ & $0 \%$ & $0 \%$ \\
\hline 10 & Medioambiente/Responsabilidad social & $3 \%$ & $0 \%$ & $0 \%$ \\
\hline 11 & SCM global & $3 \%$ & $0 \%$ & $0 \%$ \\
\hline 12 & Comportamiento del consumidor & $2 \%$ & $0 \%$ & $0 \%$ \\
\hline 13 & Gestión de Recursos Humanos & $2 \%$ & $0 \%$ & $60 \%$ \\
\hline
\end{tabular}

Fuente: Elaboración propia 
Podemos observar que las barreras de la SC están concentradas en cinco categorías temáticas: estrategia de SCM (30\%), alianzas/relaciones (30\%), e-commerce (10\%), estrategias - tiempo (20\%) y calidad (10\%). Por otro lado, en el caso de los puentes/oportunidades se observó que éstos se refieren a las siguientes 4 categorías temáticas: estrategia de SCM (20\%), tecnología de la información (10\%), desarrollo de proveedores/selección y gestión (10\%) y gestión de recursos humanos (60\%).

El gráfico demuestra también que las barreras se encuentran dentro de las categorías con mayor frecuencia de publicación. En primer lugar, las estrategias de SCM, con un 23\% de publicaciones, concentra un 30\% de las barreras. En segundo lugar, alianzas/relaciones es la tercera categoría con mayor porcentaje de publicaciones, un 16\%, y agrupa un 30\% de las barreras para el éxito de la SCM.

Además, esta comparativa nos muestra que los temas que presentan mayor relación con los puentes/oportunidades para superar estos factores críticos son los menos estudiados. Es el caso de la HRM, categoría temática que agrupa la mayor cantidad de puentes/oportunidades (60\%), pero sólo cuenta con un $2 \%$ de las publicaciones dentro del campo de la SCM en los últimos años.

Por tanto, las investigaciones en el campo de la SCM han sido dirigidas hacia el estudio de las barreras (demostrando el nivel de preocupación hacia la investigación de los factores críticos), más no así, hacia los puentes/oportunidades para alcanzar el éxito en la SCM (que son, sin duda, la clave para superar estas barreras).

Finalmente, este análisis concluye el potencial del estudio de la HRM para llenar los vacíos existentes dentro de la literatura de SCM. Estos resultados son consecuentes con las aportaciones de otros investigadores como Taylor y Taylor (2009) ${ }^{6}$, quienes establecen que la SCM sigue siendo uno de los temas de investigación predominantes y proponen como nuevo tema de investigación la importancia de la HRM.

\section{Metodología}

Una vez demostrada la importancia del estudio de la HRM en la SC, a pesar de la escasez de publica- ciones en este ámbito, se realizó un estudio bibliométrico para conocer cuáles son esas escasas investigaciones existentes, establecer su evolución a partir de la frecuencia de publicación, metodologías de investigación y principales contribuciones.

En esta dirección, para el proceso de recolección de datos se efectuó una búsqueda sistemática, haciendo uso de las siguientes bases de datos: Science Direct, Emerarld, EBSCO, Sconlit, Scopus, Inderscience, ABI/INFORM Complete y Wiley Online Library.

Para el proceso de recolección de datos se usaron palabras clave y secuencias de palabras, tales como: ( I) «gestión de la cadena de suministro» y/o «cadena de suministro», (2) «gestión de recursos humanos, recursos humanos, capital humano», entre otros, con el fin de capturar la mayor cantidad de artículos centrados en las dos disciplinas. La búsqueda se realizó en palabras claves, título, abstract y conclusiones de los artículos publicados en las bases de datos antes comentadas, sin acotar por revistas ni áreas temáticas de revistas.

A partir de los resultados de la búsqueda se localizaron un total de 46 artículos estrictamente relacionados con las dos disciplinas (RRHH y SC). Cabe destacar que fueron desestimados aquellos artículos relacionados con temáticas sobre marketing, logística u operaciones, a pesar de que en algunos casos contenían las palabras «supply chain mangement», pero quedaban alejados del objetivo de este estudio. Así, solo se incluyeron aquellos artículos que se basaban en el análisis del estudio de HRSC, y no aquellos que sólo se basaban en una parte de la cadena logística.

Se efectuó un análisis de la frecuencia de publicación de los artículos localizados sobre HRSC. Resultado de ello, se identificaron publicaciones desde el año 1997 a 2012, evidenciando que el interés de los investigadores por esta temática nació hace tan sólo quince años.

Por otro lado, se clasificaron los artículos según emplearan métodos empíricos versus no empíricos. Además, se agruparon los artículos según su contenido para la identificación de las áreas temáticas dentro del estudio de los $R R H H$, con la intención de conocer cuáles son las más estudiadas. Todo ello implicó la lectura detallada y en profundidad de los artículos identificados para la correcta designación en la te-

\footnotetext{
4 Los cuales proporcionan un registro de los temas de publicación y métodos de investigación en los 310 artículos publicados en IJOPM durante el período 2004 a 2009.
} 
mática correspondiente, así como para establecer las principales contribuciones de las investigaciones.

\section{Análisis bibliométrico de las publicaciones sobre la gestión de recursos humanos en la cadena de suministro}

En el presente trabajo se ha puesto de manifiesto la potencialidad que tiene el estudio de los HRSC, a causa del vacío existente dentro de la literatura (Giunipero et al., 2008; Shub et al., 2009; Fisher et al., 20 I 0).
Además, dado que hasta el momento no existe una revisión de la literatura sobre HRSC que dé respuesta a los interrogantes como: ¿qué ha sido mayoritariamente investigado entre estos dos campos de estudio?; ¿qué tipo de metodologías han sido utilizadas?; ¿cuáles han sido sus contribuciones y cuáles son sus carencias?, se decidió, en consecuencia, realizar un estudio bibliométrico sobre HRSC que resuelva estas incógnitas.

Los 46 artículos identificados sobre HRSC fueron localizados en tan sólo 29 revistas de diversas disciplinas (ver tabla 4), con temáticas sobre: (I) SCM, Pro-

Tabla 4

Frecuencia de publicación sobre HRSC por revistas

\begin{tabular}{|c|c|c|}
\hline & Revistas & Número de artículos por revista \\
\hline I & Human Resource Management (Special Issue) & 6 \\
\hline 2 & International Journal of Operations \& Production Management & 4 \\
\hline 3 & Supply Chain Management: An International Journal & 4 \\
\hline 4 & Organizational Dynamics & 3 \\
\hline 5 & Industrial and Commercial Training & 2 \\
\hline 6 & International Journal of Logistics Management & 2 \\
\hline 7 & International Journal of Logistics: Research and Applications & 2 \\
\hline 8 & Journal of Business Logistics & 2 \\
\hline 9 & Employee Relations & । \\
\hline 10 & Human Resource Management Journal & । \\
\hline 11 & Human Resource Management Review & I \\
\hline 12 & Human Systems Management & । \\
\hline 13 & Industrial Management \& Data Systems & । \\
\hline 14 & International Journal Integrated Supply Management & I \\
\hline 15 & International Journal of Manpower & I \\
\hline 16 & International Journal of Physical Distribution \& Logistics Management & । \\
\hline 17 & International journal of production economics & । \\
\hline 18 & International Journal of Productivity and Performance Management & । \\
\hline 19 & International Journal of Retail \& Distribution Management & I \\
\hline 20 & Journal of International Management & । \\
\hline 21 & Journal of Management Development & I \\
\hline 22 & Journal of Supply Chain Management & I \\
\hline 23 & Metalurgia International & I \\
\hline 24 & Mid-American Journal of Business & । \\
\hline 25 & MIT Center for Transportation and Logistics & 1 \\
\hline 26 & Personnel Review & I \\
\hline 27 & Quality Assurance in Education & I \\
\hline 28 & Singapore Human Resources Institute \& Curtin University of Technology & । \\
\hline & Supply Chain Management Review & I \\
\hline \multicolumn{2}{|c|}{ Total de artículos publicados } & 46 \\
\hline
\end{tabular}

Fuente: Elaboración Propia 
ducción y Logística, en un 55\% de los casos; (2) $R R H H$, con el $28 \%$ de los artículos publicados; (3) genéricas de management, en un $17 \%$.

Los artículos localizados sobre HRSC fueron publicados entre 1997 a 2012 (último año del análisis). Con la intención de valorar el comportamiento de estas publicaciones a lo largo del tiempo se realizó el análisis de frecuencias que recoge el figura I.

El artículo de mayor antigüedad fue publicado en el año 1997 y hasta 2005 las publicaciones fueron simbólicas. En estos nueve primeros años se publicaron tan sólo II artículos, lo cual representa el 24\% de los artículos. Estos datos son consecuentes con los resultados obtenidos por Giunipero et al. (2008) en su revisión de la literatura de 1996 a 2006, autores que obtuvieron unos resultados de tan sólo ocho artículos relacionados con HRSC.

Por el contrario, del 2006 al 2012 (figura 2), se observa un incremento en el número de publicaciones con un total de 35 artículos, lo cual supone el $76 \%$. Es importante destacar que en el 2010 se editó un special issue sobre Supply Chain Management por la revista Human Resource Management, lo cual supone un aumento significativo de investigaciones publicadas en este año concreto. Así, según estos resultados se aprecia en los últimos siete años un aumento del interés de los investigadores hacia el estudio de HRSC.

\section{I. Métodos de investigación en los estudios sobre HRSC}

Con la intención de evidenciar qué tipo de métodos han sido utilizados en los artículos identificados, se realizó una clasificación según emplearan métodos empíricos versus no empíricos (ver Figura 2). Esta distinción es también utilizada por Giunipero et al. (2008).

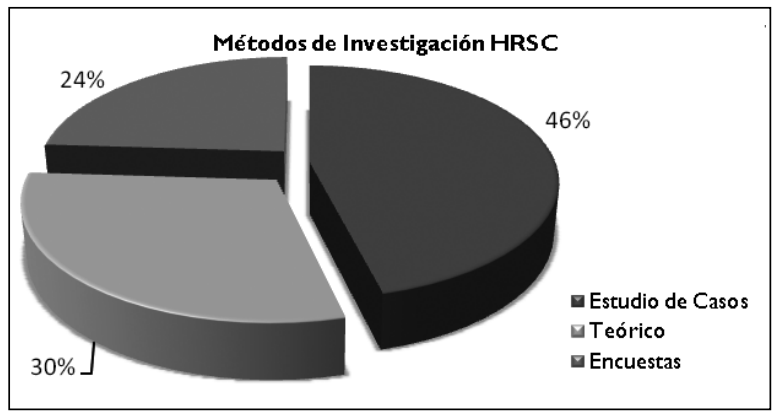

Fuente: Elaboración Propia

Figura 2

Métodos (empíricos vs. no-empíricos) utilizados en las investigaciones sobre HRSC

Los resultados indican que el estudio de casos es el método de investigación mayoritariamente utilizado por los investigadores en un $46 \%$ de los artículos, el $24 \%$ están basados en encuestas y el $30 \%$ de las publicaciones analizadas son trabajos teóricos.

El elevado porcentaje de utilización del estudio de casos como metodología de análisis frente a otras metodologías empíricas se debe probablemente a la juventud de este campo de investigación, sobre el que todavía queda camino por explorar. Dado que el estudio de casos suele emplearse para el desarrollo o contraste de una teoría o para la exploración de un fenómeno o la descripción de una situa-

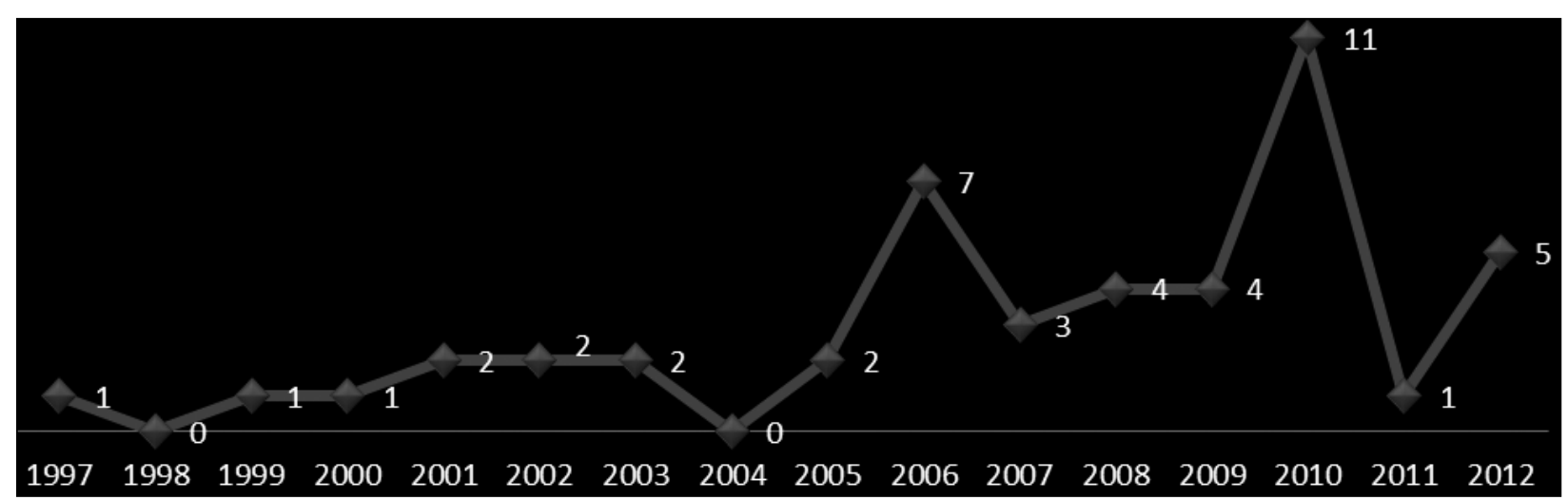

Fuente: Elaboración Propia

Figura I

Frecuencia de publicación sobre HRSC a lo largo del tiempo 
ción determinada, es una buena metodología en campos de investigación incipientes. En este sentido, según Benbasat et al. (1987), el estudio de casos se presta a la investigación exploratoria, en la que las variables son todavía desconocidas y el fenómeno no está plenamente entendido.

\section{2. Áreas temáticas abordadas en los estudios sobre HRSC}

Para conocer qué áreas temáticas se han estudiado en los trabajos publicados sobre HRSC, se efectuó una clasificación de los artículos identificados, agrupándolos por los temas más recurrentes (ver gráfico I). Las investigaciones se clasificaron en las cuatro áreas siguientes: ( I ) competencias/perfiles de los directivos y personal de apoyo de SCM, (2) impacto y mejora de la SCM a través de la HRM, (3) relaciones con los miembros de la cadena y los RRHH, (4) reclutamiento y selección de los directivos y personal de apoyo de SCM.

Así, el «impacto y mejora de la SCM a través de la $H R M \gg$ supone un 35\% de los artículos; las «competencias/perfiles de los directivos y personal de apoyo de SCM», el 35\%; las «relaciones con los miembros de la cadena y los RRHH» un I7\%; y, finalmente, el «reclutamiento y selección de los directivos y personal de apoyo de SCM»representa un I3\%.

A la vista de estos resultados, las dos principales temáticas estudiadas son: las competencias y perfiles de los directivos de la SCM y el impacto de los HRSC. A pesar de ello, los autores de los artículos analiza- dos siguen insistiendo en la necesidad de nuevos estudios en estas áreas (Rossetti et al., 2010).

\subsection{Análisis del contenido de las publicaciones sobre HRSC. Revisión de la literatura}

Tal como ya se ha comentado anteriormente, la revisión de la literatura destaca la carencia de estudios sobre HRSC evidenciado que se trata de un área olvidada de investigación (Lengnick-Hall, 20 I2). De hecho, los campos de estudio de HRSC se han tratado históricamente de forma separada, a pesar de que están «íntimamente unidos» en casi todos los entornos empresariales (Boudreau et al., 2003 en Barnes et al., 2012). Algunos autores declaran que se le ha brindado poca atención a la influencia de los RRHH en el rendimiento de la SC (Gowen, 2003). En consecuencia, a continuación se presenta el análisis de contenido de los 46 artículos identificados en el análisis bibliométrico anterior sobre HRSC, con la intención de destacar las principales contribuciones realizadas por áreas temáticas:

I) En el área de «competencias/perfiles de los directivos y personal de apoyo de la SCM» se agrupan los estudios relacionados con las competencias laborales de los puestos de la SC (los perfiles de los directivos y su personal de apoyo). En esta temática de estudio se analiza la ausencia de una única definición de SCM y que la falta de alineamiento entre la perspectiva académica y la profesional han creado cierta confusión en los departamentos de $R R H H$, especialmente en la definición de los puestos asociados con la SCM.

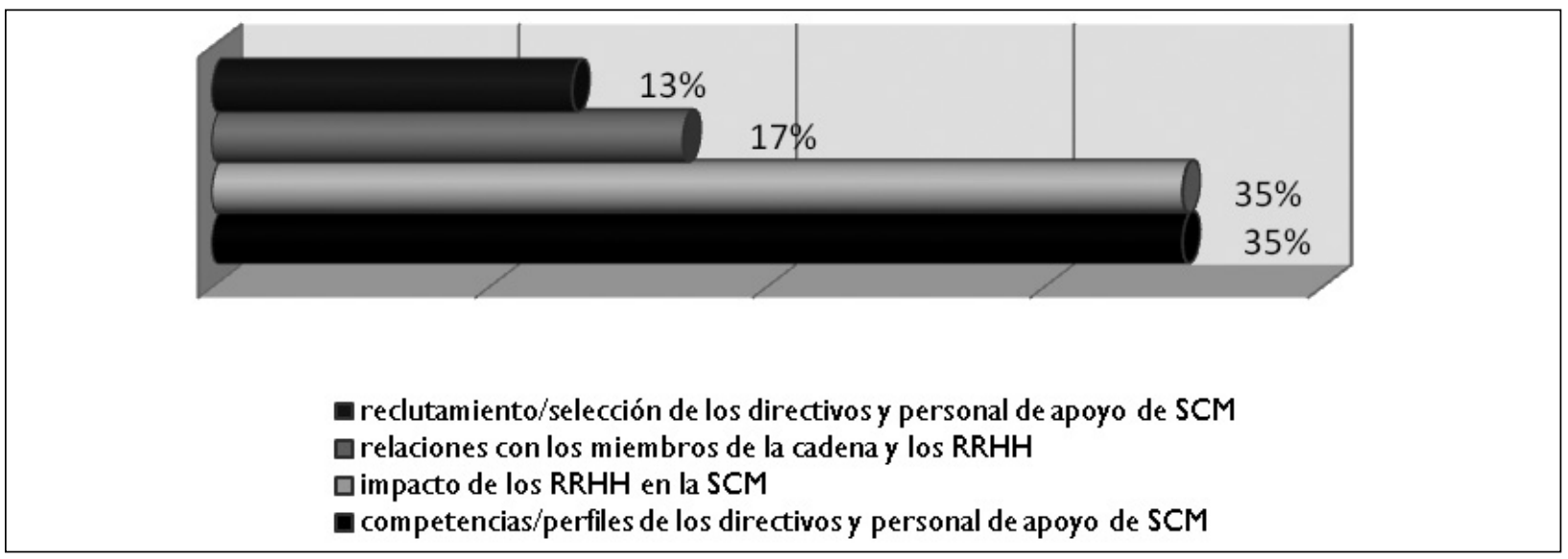

Fuente: Elaboración Propia

\section{Gráfico I}

Resultado de búsqueda de artículos publicados HRSC 